FTUV/98-58; IFIC/98-59

\title{
RADIATIVE NEUTRINO DECAY IN MEDIA
}

\author{
Dario Grasso \\ Departament de Fisica Teorica, Universitat de Valencia \\ E-46100 Burjassot - Valencia, Spain \\ E-mail: grasso@flamenco.ific.uv.es \\ and \\ Victor Semikoz \\ Institute of Terrestrial Magnetism, the Ionosfere and Radio Wave Propagation \\ Academy of Science of Russia, Troitsk, Moscow Region, 142092 Russia \\ E-mail: semikoz@izmiran.rssi.ru
}

\begin{abstract}
In this letter we introduce a new method to determine the radiative neutrino decay rate in the presence of a medium. Our approach is based on the generalisation of the optical theorem at finite temperature and density. Differently from previous works on this subject, our method allows to account for dispersive and dissipative electromagnetic properties of the medium. Some inconsistencies that are present in the literature are pointed-out and corrected here. We shortly discuss the relevance of our results for neutrino evolution in the early universe.
\end{abstract}

\section{Introduction}

Most of neutrinos in the universe are expected to be produced in the presence of environments characterised by very high temperatures and densities. In such media neutrinos behave quite differently from the way they do in vacuum [1]. The coherent interaction of neutrinos with the particles belonging to the medium can amplify or induce effects that are otherwise very feeble or absent in the vacuum. This can be crucial in order to disentangle possible anomalous properties of the neutrinos that may testify for new physics present beyond the standard model. MSW [2] neutrino resonant oscillations give a well know example of such a kind of effects.

Neutrino electromagnetic properties are also expected to be significantly affected by the presence of media. In fact, due to their coherent interaction with charged leptons and nucleons in the medium, neutrinos acquire an effective coupling to the electromagnetic field. This effect was investigated by several authors who showed how even massless neutrinos passing through the matter acquire an effective charge [3] and, in a charge asymmetric medium, also an effective magnetic dipole moment

\footnotetext{
${ }^{1}$ New address since 1st October 1998: Dipartimento di Fisica, Università di Padova, Via F. Marzolo 8, I-35131 Padova, Italy
} 
[4. Several intriguing consequences of medium induced neutrino electromagnetic couplings have been studied in the literature, e.g. plasmon decay in $\nu \bar{\nu}$ pairs [5] and Cherenkov emission of neutrinos [6].

In this letter we focus on the radiative decay of a heavy neutrino into a lighter one. Our underling particle physics model consists of a minimal extension of the standard model which allows for non-vanishing neutrino masses and mixing. In the ambit of this model, neutrino radiative decay is extremely suppressed in vacuum because of the Glashow-Iliopoulos-Maiani (GIM) cancellation [7]. However, if $T<m_{\tau}$, this is not the case in a heat-bath due to the different thermal populations of the three charged leptons families. As a consequence, the radiative neutrino decay rate in the presence of hot media exceeds the decay rate in vacuum by many order of magnitudes. This was first showed by D'Olivo, Nieves and Pal (DNP) [8] (see also [9]), who computed the decay rate both in the case of a non-relativistic plasma (NR) $\left(T \ll m_{e}\right)$ and of a ultra-relativist plasma (UR). Recently, DNP's work has been extended by Nieves and Pal [10] (NP) who showed that the decay rate may be further enhanced due to the Bose-Einstein stimulation in the production of low-energy photons $(\omega \ll T)$.

Although we agree with DNP and NP concerning the huge neutrino decay rate amplification occuring in a medium we do not, however, about their method and some of the results obtained in their works. Our main disagreement concerns the properties of the photon produced by the neutrino decay. This photon was assumed to be on the light-cone $\left(K^{2}=0\right)$ by DNP and NP. Such an assumption is equivalent to disregard any dispersive and dissipative property of photons in a thermal bath. It is well known, however, that in plasma photons behaves more like collective excitations (usually dubbed plasmons) than like free particles. While neglecting collective properties of photons may be a reasonable approximation in the high frequency regime this is however inconsistent when they are low energy or, so-called, soft. We will show that to account for the correct plasmon dispersion relation is indeed mandatory in order to avoid an unphysical behaviour of the decay rate as a function of the neutrino velocity.

Photon dissipative properties need also to be taken into account. In fact, in a heat bath low-energy photons are continuously absorbed and re-emitted mainly by Landaudamping on the heat-bath free-charges. For the first time we will show that Landaudamping gives rise to an additional, and in many cases dominant, contribution to the decay rate. Since the photon asymptotic state is a not a well defined quantity in a medium, we recourse here to a treatment that is based on finite temperature field theory and on the generalisation of the optical theorem to such a framework [11]. Within this framework the basic quantities are the Green functions which are well defined quantities even at finite temperature and density. As a bonus, one get that using such approach the role of Landau-damping comes-out naturally from the thermally-corrected photon progator [12]. A similar method has been previously applied by other authors to determine the chirality-flip rate of Dirac neutrinos in a degenerate plasma [13, 14] and in the early universe [15]. 


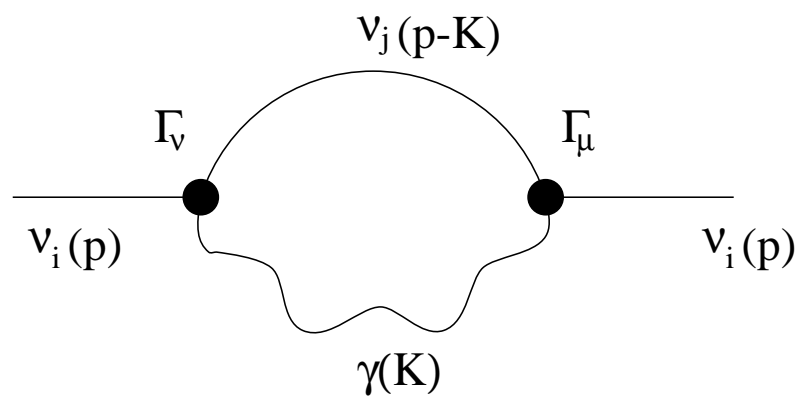

Figure 1: Feynman diagram contributing to the heavy neutrino self-energy $\Sigma$.

In this letter we only consider the case of an UR non-degenerate electron-positron plasma. As we will shortly discuss in our last section, this case is relevant for the study of neutrino evolution in the early universe. The cases in which the medium is degenerate and/or NR will be discussed elsewhere [16]. Our general method will be presented in section 2. Section 3 contains the computation of the decay rate in the case in which the plasmon is on-shell. The generalisation of this result to the case with off-shell photons is discussed in Sec. 4. Sec. 5 contains a discussion about the applications of our results to the early universe physics.

\section{The general method}

It was first showed by Weldon [11] that in a heat-bath the decay rate $\Gamma_{d}$ of a fermionic particle species (having the energy $E$ ), and the rate of the inverse process $\Gamma_{i}$, are related to the imaginary part of the fermion self-energy $\Sigma$ by the following relation

$$
\left.\operatorname{Im}\left\{\bar{u}_{(} p\right) \Sigma(p) u(p)\right\}=-E\left(\Gamma_{i}+\Gamma_{d}\right) .
$$

This result is the generalisation of the optical theorem for finite temperature and density. It is understood that $\Sigma$ includes corrections due to the effects of the medium on the field propagators.

In our case, the interesting contribution to the heavy neutrino self-energy comes from the Feynman diagram represented in Fig. 1. In that figure $\nu_{i}$ and $\nu_{j}$ are, respectively, the heavy and the light neutrino mass eigenstates participating to the decay and inverse-decay processes. Working in the real-time-formalism (RTF) we determine the imaginary part of $\Sigma$ by properly cutting 17 the diagram represented in Fig. 1. We get

$$
\operatorname{Im} \Sigma(p)=-\frac{\epsilon\left(p_{0}\right)}{2 \sin 2 \phi_{p}} \int \frac{d^{4} K}{(2 \pi)^{4}} \Gamma^{\nu *}(K)\left[S^{-}(p-K) D_{\mu \nu}^{+}(K)+S^{+}(p-K) D_{\mu \nu}^{-}(K)\right] \Gamma_{\mu}(K)
$$


where

$$
\frac{1}{2} \sin 2 \phi_{p}=\frac{e^{\beta\left|p_{0}\right| / 2}}{e^{\beta\left|p_{0}\right|}+1}
$$

and the expression for the effective vertex $\Gamma_{\mu}(K)$ will be given below. Here $K=$ $\left(k_{0}, \vec{k}\right)$ is the photon 4 -momentum in the medium rest-frame and $\epsilon\left(p_{0}\right)$ is the sign function. We observe that a positive $k_{0}$ accounts for the process with emission of a photon (energy given to the medium) whereas a negative $k_{0}$ takes care of the inverse process (energy lost by the medium). We are then able to disentangle the different contributions to $\Gamma_{d}$ and $\Gamma_{i}$ from (11) by properly choosing the $k_{0}$ integration interval. For the sake of simplicity we assume the light neutrino to be massless. In the RTF the off-diagonals component of its propagator are

$$
S^{ \pm}(p-K)=-2 \pi i(\hat{p}-\hat{K})\left(\theta\left( \pm\left(p_{0}-k_{0}\right)-n_{F}\left(\left|p_{0}-k_{0}\right|\right)\right) \delta\left((p-K)^{2}\right)\right.
$$

where $n_{F(B)}$ is the Fermi-Dirac (Bose-Einstein) distribution function. In the rest frame of the medium (and in the Landau gauge) the photon propagator can be decomposed into

$$
D_{\mu \nu}^{ \pm}(K)=D_{T}^{ \pm} P_{\mu \nu}(K)+D_{L}^{ \pm} Q_{\mu \nu}(K) .
$$

The transverse and longitudinal projector operators are defined by

$$
P_{\mu \nu}(K)=\left(\delta_{i j}-\frac{k_{i} k_{j}}{k^{2}}\right) \delta_{\mu i} \delta_{\nu j} \quad Q_{\mu \nu}(K)=e_{\mu}(K) e_{\nu}(K)
$$

where $e_{\mu}(K)=\left(k, k_{0} \vec{k} / k\right) / \sqrt{K^{2}}$ is the polarization versor. The coefficients $D_{T, L}^{ \pm}$are given by [12, 18]

$$
\begin{aligned}
& D_{T, L}^{ \pm}=2\left(\theta ( \pm k _ { 0 } - n _ { B } ( | k _ { 0 } | ) ) \left[\quad \pi \delta\left(K^{2}-\operatorname{Re} \Pi_{T, L}(K)\right) \delta_{K r}\left(\operatorname{Im} \Pi_{T, L}(K)\right)\right.\right. \\
& \left.-\frac{\operatorname{Im} \Pi_{T, L}(K)}{\left(K^{2}-\operatorname{Re} \Pi_{T, L}(K)\right)^{2}+\left(\operatorname{Im} \Pi_{T, L}(K)\right)^{2}}\right]
\end{aligned}
$$

where $\delta_{K r}$ is a Kroneker function. These expressions include the one-loop thermal corrections to the photon propagator. It is known [19] that for soft photons $\left(k_{0}, k \lesssim e T\right)$ these corrections cannot be neglected and that a meaningful perturbative expansion can be obtained performing a proper resummation of the hard-thermal-loops contributing to the photon polarization tensor.

Equation (7) has been written in such a way in order to distinguish an on-shell part, corresponding to the propagation of the transversal and longitudinal modes of a plasmon, and an off-shell part associated to Landau-damping. The fluctuationdissipation theorem shed light on the physical nature of off-shell plasmons: they are thermal fluctuations of the electromagnetic field [18]. As suggested by the BreitWigner form of the off-shell part of $D_{T, L}^{ \pm}$, we can think of electromagnetic thermal 
fluctuations as resonances which are continuously emitted and re-absorbed from the free-charges in the plasma by Cherenkov and inverse-Cherenkov emission.

The general expression of the polarization tensor in a isotropic plasma is

$$
\Pi_{\mu \nu}(K)=\Pi_{T}(K) P_{\mu \nu}(K)+\Pi_{L}(K) Q_{\mu \nu}(K)
$$

where, for an UR plasma, we have [20]

$$
\begin{aligned}
& \operatorname{Re} \Pi_{T}(K)=\frac{3 \omega_{P}^{2}}{2}\left[\frac{k_{0}^{2}}{k^{2}}+\left(1-\frac{k_{0}^{2}}{k^{2}}\right) \frac{k_{0}}{2 k} \ln \left|\frac{k_{0}+k}{k_{0}-k}\right|\right], \\
& \operatorname{Re} \Pi_{L}(K)=3 \omega_{P}^{2}\left(1-\frac{k_{0}^{2}}{k^{2}}\right)\left[1-\frac{k_{0}}{2 k} \ln \left|\frac{k_{0}+k}{k_{0}-k}\right|\right]
\end{aligned}
$$

and

$$
\begin{aligned}
& \operatorname{Im} \Pi_{T}(K)=-\frac{3 \omega_{P}^{2}}{2} \pi\left(1-\frac{k_{0}^{2}}{k^{2}}\right) \frac{k_{0}}{2 k} \theta\left(k^{2}-k_{0}^{2}\right), \\
& \operatorname{Im} \Pi_{L}(K)=3 \omega_{P}^{2} \pi\left(1-\frac{k_{0}^{2}}{k^{2}}\right) \frac{k_{0}}{2 k} \theta\left(k^{2}-k_{0}^{2}\right) .
\end{aligned}
$$

In the UR limit, the plasma frequency is given by $\omega_{P}=e T / 3$.

Plasmon dispersion relations are determined by solving $K^{2}=\operatorname{Re} \Pi_{T, L}(K)$. Analytic forms of the solutions can be obtained in some suitable limits 12 . In the $k \rightarrow 0$ limit transversal and longitudinal dispersion relations are respectively

$$
\begin{array}{ll}
k_{0}^{2}=\omega_{P}^{2}+\frac{6}{5} k^{2} & \text { transversal }, \\
k_{0}^{2}=\omega_{P}^{2}+\frac{3}{5} k^{2} & \text { longitudinal },
\end{array}
$$

whereas in the hard limit $\left(k_{0}, k \gg \omega_{P}\right)$ one gets

$$
\begin{array}{ll}
k_{0}^{2}=\frac{3}{2} \omega_{P}^{2}+k^{2} & \text { transversal }, \\
k_{0}=k^{2}+4 k^{2} e^{-2 k^{2} / 3 \omega_{P}} & \text { longitudinal . }
\end{array}
$$

\section{The on-shell plasmon case}

We now come into some more details of our computation. As we discussed in the introduction, in a medium the neutrino-photon coupling is mediated by the weak interaction of the neutrino to the free charges in the plasma. We the sake of simplicity we only consider here a non-degenerate electron-positron plasma at temperatures below the muon mass. From [3, 21] we know that the effective medium induced neutrino electromagnetic vertex is given by

$$
\Gamma_{\mu}(K)=U_{i e} \frac{G_{F}}{e} \Pi_{\mu \nu}(K) \gamma^{\nu} L
$$


where $U$ is the lepton mixing matrix and $L \equiv \frac{1}{2}\left(1-\gamma_{5}\right)$.

By substituting (13), (14) and the on-shell part of (7) in (2), we find that the decay rate of the heavy neutrino into the lighter one and a transversal plasmon is

$$
\begin{aligned}
\Gamma_{T}^{o n}=\frac{1}{2 \sin 2 \phi_{p}} \quad & \frac{G_{F}^{2}}{4 E e^{2}}\left|U_{i e}^{*} U_{j e}\right|^{2} \int \frac{d^{4} K}{(2 \pi)^{4}}\left(\operatorname{Re} \Pi_{T}(K)\right)^{2} \delta\left(K^{2}-\operatorname{Re} \Pi_{T}(K)\right) \delta\left((p-K)^{2}\right) \\
& \times\left[\epsilon\left(p_{0}-k_{0}\right)\left(\theta\left(-k_{0}\right)+n_{F}\left(\left|p_{0}-k_{0}\right|\right)\right)+\epsilon\left(p_{0}-k_{0}\right)\left(\theta\left(k_{0}\right)+n_{B}\left(\left|k_{0}\right|\right)\right)\right] \\
& \times P_{\alpha \beta} \operatorname{Tr}\left\{(\hat{p}-m) \gamma^{\beta}(\hat{p}-\hat{K}) \gamma^{\alpha} L\right\}
\end{aligned}
$$

where $m$ is the heavy neutrino mass. In the case the plasmon is longitudinal the rate can be obtained by replacing in (14) the subscript $T$ with $L$ and the projector $P_{\alpha \beta}$ with $Q_{\alpha \beta}$. In the square brackets in the right side of (14) we can distinguish a term accounting for the Bose-Einstein stimulated photon emission and a Pauli-blocking term for the production of the light neutrino. Since generally the latter is much smaller than the former we disregard the Pauli-blocking term in the following. We also approximate the factor $\frac{1}{\sin 2 \phi_{p}}$ with the unity.

Using $\delta\left((p-K)^{2}\right)$ and $\delta\left(K^{2}-\operatorname{Re} \Pi_{T}(K)\right)$ to suppress respectively the angular and the temporal parts of the $\frac{d^{4} K}{(2 \pi)^{4}}$ integration we get

$$
\Gamma_{T}^{o n}=\frac{9 G_{F}^{2}}{32 \pi e^{2}}\left|U_{i e}^{*} U_{j e}\right|^{2} \omega_{P}^{5} f_{T}(v) .
$$

Here $v$ is the velocity of the heavy neutrino with respect to the medium rest frame. Since it is in general impossible to find an analytical form of the plasmon dispersion relation, it is convenient to distinguish between a soft and a hard contribution to (15). In the case the plasmon is transversal we have

$$
\begin{gathered}
f_{T}(v)=f_{T}^{\text {hard }}(v)+f_{T}^{\text {soft }}(v) \\
f_{T}^{\text {soft }}(v) \simeq \frac{8}{9} \frac{1-v^{2}}{\tilde{m}^{2} v} \int_{\tilde{k}_{m i n}^{\text {soft }}}^{\tilde{k}_{\text {soft }}^{\text {soft }}} d \tilde{k}\left(1+n_{B}(1)\right)\left\{\tilde{E}^{2}-\frac{1}{2}\left(1+\tilde{m}^{2}\right)-\frac{1}{\tilde{k}^{2}}\left[\tilde{E}-\frac{1}{2}\left(1+\tilde{m}^{2}\right)\right]\right\} \theta(1-\tilde{k})
\end{gathered}
$$

and

$$
f_{T}^{\mathrm{hard}}(v) \simeq \frac{1-v^{2}}{\tilde{m}^{2} v} \int_{\tilde{k}_{m i n}^{\mathrm{hard}}}^{\tilde{k}_{\text {har }}^{\mathrm{hard}}} \frac{d \tilde{k}}{\tilde{k}}\left(1+n_{B}(\tilde{k})\right)\left\{\tilde{E}-\frac{\tilde{k}^{2}}{2}-\frac{\tilde{m}^{2}}{4 \tilde{k}}\right\} \theta(\tilde{k}-1)
$$

where $E$ is the energy of the heavy neutrino and tilted quantities have been normalised to $\omega_{P}$ so that $f_{T}(v)$ is a dimensionless function. The integration limits are

$$
\tilde{k}_{\max }^{\mathrm{hard}}=\frac{\tilde{m}}{2} \sqrt{\frac{1+v^{2}}{1-v^{2}}} \quad \tilde{k}_{\min }^{\mathrm{hard}}=\frac{\tilde{m}}{2} \sqrt{\frac{1-v^{2}}{1+v^{2}}},
$$




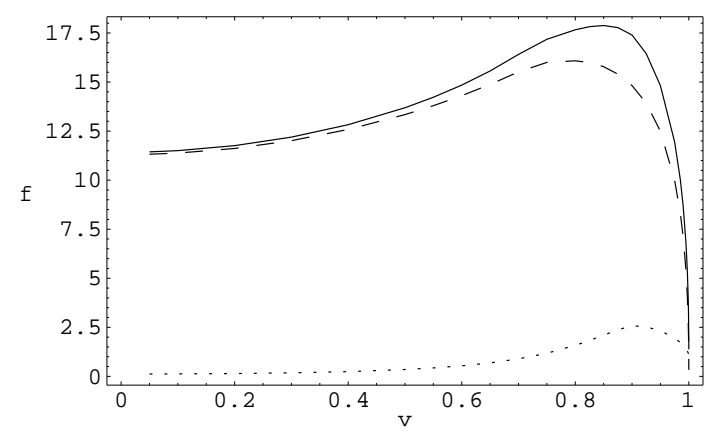

Figure 2: In this figure the dashed, dotted and continuous lines represents respectively the functions $f_{T}^{\text {hard }}(v), f_{T}^{\text {soft }}(v)$ and the sum of the two. Here we fixed $\tilde{m}=5$.

in the hard limit, and

$$
\tilde{k}_{\max }^{\mathrm{soft}}=\tilde{m} \sqrt{\frac{1+v^{2}}{1-v^{2}}}-1 \quad \tilde{k}_{\min }^{\mathrm{soft}}=\left|\tilde{m} \sqrt{\frac{1-v^{2}}{1+v^{2}}}-1\right|,
$$

in the soft one. For reason of available space, only a zero order expansion in $\tilde{k}$ of $\operatorname{Re} \Pi_{T}(K)$ has been reported in the expressions (18) and (17). However, a more complete second order expansion has been used to get Fig. 2. Also, in our numerical integrations some more suitable exponential cut-off functions have been used instead of the step functions appearing in the integrals in (18) and (17). From Fig. 2 we see that in spite of the large enhancement of the Bose-Einstein factor $n_{B}\left(\left|k_{0}\right|\right)=$ $\left(e^{\beta\left|k_{0}\right|}-1\right)^{-1} \simeq T / k_{0} \gg 1$ that one gains in the soft limit, the soft contribution to the total decay rate is subdominant with respect to the hard one. This is due to the smaller available phase space when the plasmon is soft. Not surprisily, such a situation becomes even more pronounced for higher values of the decaying neutrino mass. The maximum value of $f_{T}^{\text {soft }}$ is reached at the velocity $v_{*} \equiv \frac{m^{2}-\omega_{P}^{2}}{m^{2}+\omega_{P}^{2}}$ where $\tilde{k}_{\text {min }}^{\text {soft }}=0$.

It is worthwhile to observe that, a part for the presence of the infra-red cut-off function (and of an extra factor of 2), our expression (18) coincides with the result found by NP. In our opinion, the introduction of the cut-off is essential in order to avoid the incorrect use of the hard plasmon dispersion relation $\left(K^{2} \simeq 0\right)$ in the soft limit. In fact, in the limit in which the decaying neutrino is ultra-relativistic $(v \rightarrow 1)$ it is evident from (19) that $\tilde{k}_{\text {min }}^{\text {hard }} \rightarrow 0$. As we have seen above, our approach solves such a problem by properly separating the hard and the soft contributions to $f_{T}(v)$. To confirm us of the validity of our method, we get a physically correct behaviour of the decay rate that drops to zero when $v \rightarrow 1$ (see Fig. 2). Such a result is actually 
convincing since no rest frame is available for the neutrino to decay in when it travels at the speed of light. A non-vanishing rate was instead found by NP in the limit $v \rightarrow 1$.

In the case the plasmon is longitudinal only the soft term contributes to the total decay rate. In fact, as we can see from the second of the equations (12) $K^{2}$, hence also $\operatorname{Re} \Pi_{L}(K)$, vanishes in the hard limit. Due to angular momentum conservation, $\Gamma_{L}(K)$ is suppressed by a factor $\left(\omega_{P} / m\right)^{2}$ with respect to the soft contribution to $\Gamma_{T}(K)$. For this reason its contribution to the total decay rate is subdominant. For reason of available space the expression of $\Gamma_{L}(K)$ will be presented elsewhere [16].

\section{The off-shell plasmon case}

In this section we discuss the contribution of thermal fluctuations of the electromagnetic field to the neutrino decay. As we discussed in the Sec. 2 the effect of these fluctuations is accounted by the second term on the right side of (7). Differently from the case considered in the previous section, plasmons associated to thermal fluctuations are not on-shell hence they do not obey any dispersion relation. As a consequence, in these case we have to deal with a double integration both over $k$ and $k_{0}$. Indeed, the expressions for the decay rate look in this case

$$
\Gamma_{T, L}^{o f f}=\frac{9 G_{F}^{2}}{32 \pi e^{2}}\left|U_{i e}^{*} U_{j e}\right|^{2} \omega_{P}^{5} g_{T, L}(v)
$$

The expression of the kinematical functions $g_{T, L}(v)$ are

$$
\begin{aligned}
g_{T}(v) \simeq 2 \tilde{T} \frac{1-v^{2}}{\tilde{m}^{2} v} \int_{0}^{\infty} d \tilde{k}_{0} \int_{\tilde{k}_{m i n}^{\text {soft }}}^{\tilde{k}_{m a x}^{\text {soft }}} \frac{d \tilde{k}}{\tilde{k}^{2}} & \\
\times & {\left[\tilde{E}^{2}-\frac{\left(\tilde{K}^{2}+\tilde{m}^{2}\right)}{2}-\frac{\left(\tilde{E} \tilde{k}_{0}-\frac{1}{2}\left(\tilde{K}^{2}+\tilde{m}^{2}\right)\right)^{2}}{\tilde{k}^{2}}\right] }
\end{aligned}
$$

and

$$
\begin{aligned}
g_{L}(v) \simeq \frac{\tilde{T}}{2} \frac{1-v^{2}}{\tilde{m}^{2} v} \int_{0}^{\infty} d \tilde{k}_{0} \int_{\tilde{k}_{\min }^{\mathrm{soft}}}^{\tilde{k}_{\text {sox }}^{\mathrm{soft}}} \frac{d \tilde{k}}{\tilde{k}^{4}} & {\left[\left(2 \tilde{E}-\tilde{k}_{0}\left(1+\frac{\tilde{m}^{2}}{\tilde{K}^{2}}\right)\right)^{2}-\left(\tilde{K}^{2}-\tilde{m}^{2}\right) \tilde{k}^{2}\right] } \\
\times & \tilde{K}^{4} A_{L}\left(\tilde{k}_{0}, \tilde{k}\right) \theta\left(\tilde{k}^{2}-\tilde{k}_{0}^{2}\right)
\end{aligned}
$$

where

$$
A_{T, L}\left(\tilde{k}_{0}, \tilde{k}\right) \equiv \frac{\left(\operatorname{Re} \Pi_{T, L}(K)\right)^{2}+\left(\operatorname{Im} \Pi_{T, L}(K)\right)^{2}}{\left(K^{2}-\operatorname{Re} \Pi_{T, L}(K)\right)^{2}+\left(\operatorname{Im} \Pi_{T, L}(K)\right)^{2}} .
$$

Since Landau-damping is effective only for soft photons we can neglect here any contribution coming from the hard part of the photon spectrum. This allowed us to perform the substitution $\left(1+n_{B}\left(k_{0}\right)\right) \rightarrow T / k_{0}$ in (22,23). The functions $g_{T, L}(v)$ have 


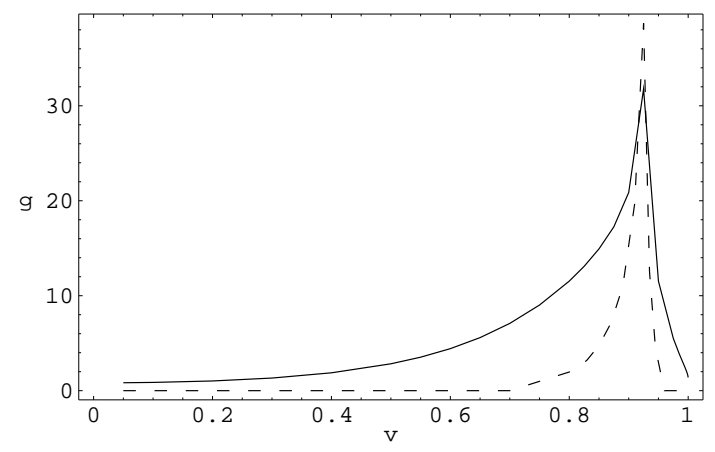

Figure 3: In this figure the dashed and the continuous lines represents respectively the functions $g_{T}(v), g_{L}(v)$. Again, we fixed here $\tilde{m}=5$.

been computed numerically and their plots are reported in Fig. 3. From this figure we see that like for the soft contribution to $\Gamma_{T, L}^{o n}, \Gamma_{T, L}^{o f f}$ reach their common maximum value when $v=v_{*}$. This is not unexpected since the functions $A_{T, L}(K)$ take their maximum values when $K^{2}$ approaches $\operatorname{Re} \Pi_{T, L}(K)$.

We have, however, a crucial difference between the off-shell and the on-shell contributions to the total decay rate. Whereas radiative neutrino decay is kinematically forbidden if $K^{2}>m^{2}$ this is clearly not the case whenever the photon is off-shell. Note that even in the hard limit, where $K^{2} \simeq \frac{3}{2} \omega_{P}^{2}$ (see (12)), the on-shell decay cannot take place unless $m^{2}>\frac{3}{2} \omega_{P}^{2}$. This was not noted in [8, 9, 10]. We have to mention, however, that although $\Gamma^{o f f}$ remains different from zero it is typically very small when $m \ll \omega_{P}$.

\section{Discussion}

The results of our previous sections can be directly applied to study neutrino evolution in the early universe where, for $T \gg 1 \mathrm{MeV}$, the electron-positron plasma is UR and non-degenerate. For definitess we consider the radiative decay of a tau-neutrino with a mass in the $\mathrm{MeV}$ range into a massless $\nu_{e}$. In order to determine if radiative decay can give rise to any relevant depletion of tau-neutrinos we have to compare the decay rate with the expansion rate of the universe. Using our previous results we find that the decay rate is given by

$$
\Gamma \simeq 2 \times 10^{-5}\left(\frac{T}{1 \mathrm{MeV}}\right)^{5}\left|\sin ^{2} 2 \theta\right| \frac{f(v)+g(v)}{10} s^{-1}
$$


where $\theta$ is the vacuum mixing angle between the $\nu_{e}$ and the $\nu_{\tau}$ and $f(v)$ and $g(v)$ are the sum of the transversal and longitudinal kinematical functions computed in the previous sections. As we showed above $f(v)+g(v)$ is typically of the order of few tens if $m \approx T$. At the big-bang nucleosynthesis $(\mathrm{BBN})$ time the Hubble rate is given by

$$
H \simeq 1\left(\frac{T}{1 \mathrm{MeV}}\right)^{2} s^{-1}
$$

It is easy to verify that $\Gamma$ can exceed $H$ only for temperatures that are above (though not too much) the range at which BBN take place $\left(0.1 \lesssim T_{B B N} \lesssim 10 \mathrm{MeV}\right)$. Furthermore, even if $\Gamma>H$ at such high temperatures, one should not forget that no neutrino depletion could take place since, for $T \gtrsim 2 m$, standard weak processes are always able to re-produce tau-neutrinos.

A different scenario might be realised in the case one considers a larger extension of the standard model which allows for the presence of a neutrino transitional magnetic moment. A magnetic moment induced decay can become dominant with respect to that induced by the medium when the plasma in NR and neutrinos are out of thermal equilibrium. Although Landau-damping gives an extra contribution to the decay also in this case, we do not expect that this effect could give rise to a significant neutrino depletion after BBN. We observe, however, that astrophysical and cosmological constraints on the neutrino transitional magnetic moments, that have been previously derived disregarding dispersive and dissipative properties of the medium, should be reconsidered in the light of the results obtained in this work. This, as other related issues, will be discussed in a forthcoming paper [16].

\section{Acknowledgements}

The work of D.G. have been supported by the TMR network grant ERBFMRXCT960090 of the European Union, and that of V.S. by INTAS grant 96-0659 and by the sabbatical grant SAB95-506 and RFFR 97-02-16501, 95-02-03724. 


\section{References}

[1] For a wide review about neutrino properties in media see: G. Raffelt, Stars as Laboratories for Fundamental Physics, The University of Chicago Press, Chicago, 1996.

[2] L. Wolfenstein, Phys. Rev. D17 (1978) 2369; S.P. Mikheyev and A.Y. Smirnov, Nuov. Cim. C9 (1986) 17.

[3] V.N. Oraevsky and V.B. Semikoz, Physica A 142 (1987) 135; J.F. Nieves and P.B. Pal, Phys. Rev. D49 (1994) 1398.

[4] V.B. Semikoz, Sov. J. Nucl. Phys. 46 (1987) 946.

[5] D. Grasso and E.W. Kolb, Phys. Rev. D48 (1993) 3522; T. Altherr and P. Salati, Nucl. Phys. B421 (1994) 662.

[6] V. Semikoz and Ya.A. Smorodinsky, Sov. Phys. JETP 68 (1989) 20; J.C. D'Olivo, J.F. Nieves and P.B. Pal, Nucl. Phys. Proc. Suppl. 48 (1995), 269.

[7] P.B. Pal and L. Wolfenstein, Phys. Rev. D25 (1982) 766.

[8] J.C. D'Olivo, J.F. Nieves and P.B. Pal, Phys. Rev. Lett. 64 (1990) 1088.

[9] C. Giunti, C.W. Kim and W.P. Lam, Phys. Rev. D43 (1991) 164.

[10] J.F. Nieves and P.B. Pal, Phys. Rev. D56 (1997) 365.

[11] H. A. Weldon, Phys. Rev. D28 (1983) 2007.

[12] T. Altherr, Ann. Phys. (N.Y.) 207 (1991) 374.

[13] T. Altherr and K. Kainulainen, Phys. Lett. B262 (1991) 79.

[14] A. Ayala, J.C. D'Olivo and M. Torres, hep-ph/9804230.

[15] P. Elmfors, K. Enqvist, G. Raffelt and G. Sigl, Nucl. Phys. B503 (1997) 3.

[16] D. Grasso and V. Semikoz, work in progress.

[17] R. Kobes and G. W. Semenoff, Nucl. Phys. B260 (1985) 714.

[18] D. Lemoine, Phys. Rev. D51 (1995) 2677.

[19] E. Braaten and R. D. Pisarski, Nucl. Phys. B337 (1990) 569.

[20] H. A. Weldon, Phys. Rev. D26 (1982) 1394.

[21] K. Enqvist, K. Kainulainen and V. Semikoz, Nucl. Phys. B374 (1992) 392. 